\title{
Stress Visualisation in Gastrointestinal Tract at Different Pathologies Forwards a Systems Approach to Drug Repurposing
}

\section{Rao VL Papineni ${ }^{1,2,3 *}$, Shahid Umar ${ }^{1}$, James Bown ${ }^{4}$ and Alexey Goltsov ${ }^{4 *}$}

${ }^{1}$ University of Kansas Medical Centre, KUMC, USA

${ }^{2}$ Precision X-Ray Inc. North Branford, USA

${ }^{3} P A C T$ and Health, Branford, USA

${ }^{4}$ School of Science, Engineering and Technology, Abertay University, Dundee, UK

Novel non-invasive bio photonic techniques for deep-tissue imaging yield valuable information on the physiological processes occurring at cellular and organ scales in small animals. Bio photonic tools applying fluorescence and bioluminescence imaging provides researchers with new opportunities to visualise in $3 \mathrm{D}$ and in real time angiogenesis, brain activity, tumour development and its vascularisation [1] and radiation injuries responsive radio bioluminescence [2]. Development of new transgenic mice with transcription factor activity driven genetic reporting opens up new facets in genetic and signalling networks in vivo [3]. An emerging trend with these new developments is to visualize responses of a living organism to exposure to different pathogens, stresses and diseases. For example, in vivo visualisation of oxidative stress shows that excessive production of reactive oxygen species (ROS) is implicated in an early phase of different pathologies and physiological states [4-7].

Real time visualisation of ROS activity (Figures 1A and 1B) under different pathological conditions shows a robust increase in the gastrointestinal tract region during radiation injuries and Citrobacter rodentium intestinal infection $[4,5]$. ROS activity determination was facilitated using a sensitive cooled CCD camera and a chemiluminescence molecular probe. Thus, the host's inflammatory response in terms of direct formation of ROS and subsequent oxidative stress is similar to GI injury resulting either from whole body $\mathrm{X}$-ray irradiation or following infection with an enteric pathogen. This marked resemblance can be similarly extended to other pathophysiological conditions. Using an ovarian metastasis mouse cancer model (Figure 1C), ROS activity (red colour) was observed to be a familiar player in putative metastasis (green colour) formation [6,7]. Oxidative stress from the different pathological processes noted above responds and converges on ROS up regulation and induction of oxidative stress response, and various cells within the abdominal microenvironment are involved. There is a close cooperation (Figure 2) and cross talk among GI epithelia cells, intestinal stem cells, macrophages, GI micro biota and vagal sensory neurons, which are involved in oxidative stress response to bacterial pathogen at the cellular and organism levels.

ROS up regulation is closely linked to inflammation, which is in turn associated with immunity activation and up regulation of pro-
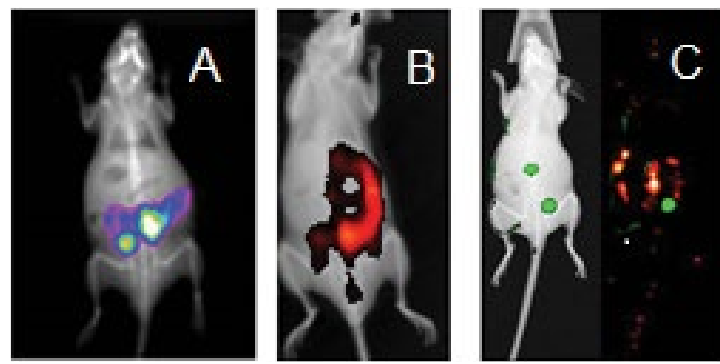

Figure 1: (A) Visualisation of oxidative stress induced by X-ray irradiation, (B) Citrobacter rodentium intestinal infection (C) tumour progression [4-7]. inflammatory cytokines, chemokines, growth factors, TNFa and formation of inflammasomes. Inflammation is a highly regulated pathway, and is mediated by pro- and anti-inflammatory biochemical signals. The main source of ROS in the GI microenvironment is reported to be NADPH oxidases (NOX1-5) and mitochondria in macrophages and other cells that generate the superoxide anion in phagosome or the extracellular environment [8]. Intriguingly, recent data suggest both up- and down-regulation of NOX activity may cause inflammatory complications [8]. ROS is likely to be a key signalling event in inflammation development and termination, and functions as a pro- and anti-inflammatory messenger in multiple signal and metabolic pathways through oxidative modulation of redox-sensitive proteins bearing thiol groups. One of the critical signalling involving ROS is T-cell activation by ROS generated by macrophages.

Another effect of ROS production in conjunction with inflammation signalling and immune system response is suggested to be a change in

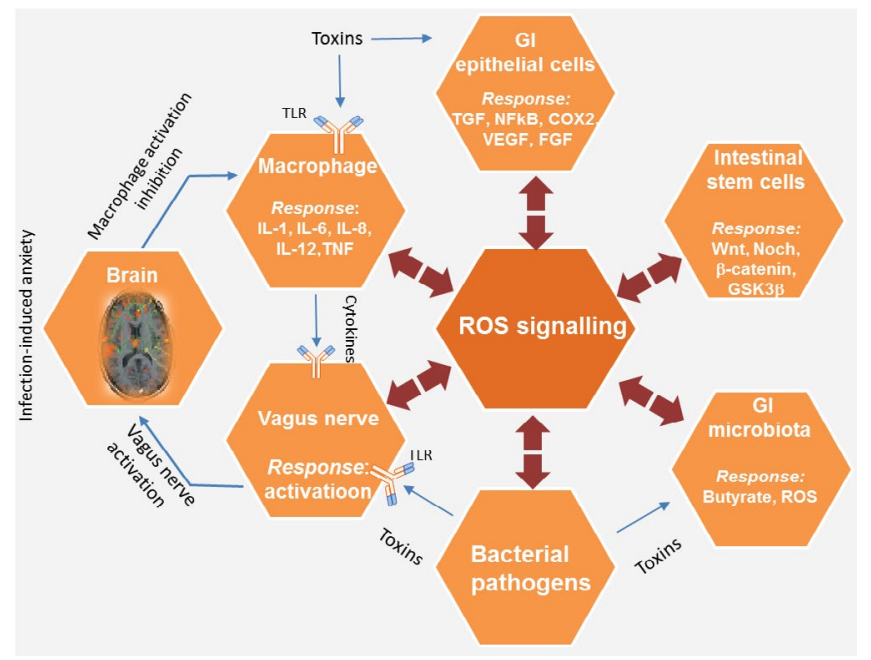

Figure 2: Inflammation response of GI tract cells to bacterial pathogen and cell cross talk via ROS signalling.

*Corresponding author: Alexey Goltsov, School of Science, Engineering and Technology, Abertay University, Dundee, UK, Tel: +44-1382-308-432; E-mail: A.Goltsov@abertay.ac.uk

Rao VL Papineni, PACT \& Health, Branford, USA, Tel: +1-713-799-2661; E-mail: papineni@graduate.hku.hk

Received July 14, 2015; Accepted July 15, 2015; Published July 18, 2015

Citation: Papineni RVL, Umar S, Bown J, Goltsov A (2015) Stress Visualisation in Gastrointestinal Tract at Different Pathologies Forwards a Systems Approach to Drug Repurposing. J Bioanal Biomed 7: e129. doi:10.4172/1948-593X.1000e129

Copyright: ( $) 2015$ Papineni RVL, et al. This is an open-access article distributed under the terms of the Creative Commons Attribution License, which permits unrestricted use, distribution, and reproduction in any medium, provided the original author and source are credited. 
the regenerative response of intestinal stem cells (ISCs) or progenitors [9] (Figure 2). It is possible that ISC might respond to GI injury, DNA damage response signalling, cytokine and ROS released by macrophage at irradiation, and other pathogen agents [10]. This signalling is proposed to activate fast regeneration of GI tract tissues following GI damage.

Another key role of ROS signalling is in the modulation of hostpathogen interactions and the gut-microbiota-host immune system interaction. The microbiota that inhabits the mammalian intestine can modulate a range of physiological functions, and includes control over immune responses, epithelial barrier function, and cellular proliferation. Dysbiosis is a gastro-intestinal perturbation that can be induced by the administration of antibiotics, indiscreet dietary practices, medications, immune deficiencies, inflammation and pathogenic infections. Dysbiosis can lead to increased numbers of Gram-negative bacteria and translocation of their gene products such as lipopolysaccharides (LPS) across the inflamed, permeable intestinal epithelia generating systemic inflammation and endotoxemia $[11,12]$. It has been has shown recently that gut epithelia following contact with commensal bacteria rapidly generate ROS [12]. Biophotonic tools have dissected the micro biome role in ROS production more cogently during radiation injuries [13]. ROS production in professional phagocytes is generally induced via stimulation of formyl peptide receptors (FPRs) and activation of Nox2 in addition to its generation in other cell types, including intestinal epithelia, in response to microbial signals via FPRs and epithelial NOX1. ROS generated by NOX enzymes has been shown to function as a critical second messenger in multiple signal transduction pathways via the rapid and transient oxidative inactivation of a distinct class of sensor proteins bearing oxidant-sensitive thiol groups. These redoxsensitive proteins include tyrosine phosphatases that serve as regulators of MAP kinase pathways, focal adhesion kinase, as well as components involved in NF- $\mathrm{\kappa B}$ activation. The microbe-elicited ROS can stimulate cellular proliferation and motility as well as modulate innate immune signalling [11].

More recently, ROS has been implicated in the development of colonies and biofilms that represent microbial multicellularity. Some cohorts of human gastrointestinal bacteria were reported to be able to generate a rapid increase in ROS in response to harmful stimuli via activation of epithelial NOX1 [12]. Gastrointestinal dysbiosis accompanying X-ray irradiation of mice (Figure 1A) suggests that alteration in gut bacteria may precede inflammatory processes. Interestingly, following $C$. rodentium infection, mice develop colitis and this causes a pronounced dysbiosis that is characterized by an overgrowth of $C$. rodentium and a consequent reduction in the abundance and overall diversity of the resident microbiota.

Another physiological system that strictly controls immune response to GI injury is the central nervous system. The autonomic surveillance function of the vagus nervous cells in the submucosa region is due to the expression of Toll-like receptors (TLRs) and receptors for cytokines, and TNF that allow the sensory neurons respond to the pathogen presence or GI damage independent of immune activation and contribute to activation of immune system in part via the regulation of lymphocyte recruitment [14]. It is now established that the vagus nerve forms the immune-GI-brain circuitry which controls both pro- and anti-inflammatory processes. Intriguingly, the role of vagus nerve signalling extends to human mood modulation and behaviour: it was found that this signalling activates the region in the brain responsible for fear and anxiety following GI injury [15]. This immune-GI-brain communication axis is the arena of study for the fields of psychoneuroimmunology and immunobehavioral research, which originated from sickness symptoms, related to bacterial infection but is recognized to expand spectrum of diseases ranging from cancer to Type 1 Diabetes.

The imaging of the GI tract region with increasing ROS in cancer mouse models (Figure 1C) shows a similar genotoxic stress response of GI microenvironment to cancerous lesions, and this suggests a link between inflammatory/immunity and cancerogenesis. The cells depicted in Figure 2 along with other cells that form the tumour microenvironment (TME) are enriched with pro-inflammatory cytokines IL- $1 \beta$, TNF- $\alpha$, INF- $\gamma$, and CCL2 promoting cancer development and invasion and defending cancer cells from host immunity, defining anticancer therapeutic resistance, and making up an environment for cancer stem cells [16]. The role of inflammation, immune system and ROS in a chronically inflamed TME is currently the subject of in-depth research. Results so far are controversial, showing the dual roles of immune response in GI cancer [16]. Inflammation has been shown to contribute to both tumour development and antitumor immunity. Most human tumours are associated with diverse immune cell infiltrates; in part macrophages, and the role and impact of extreme levels of ROS and macrophage activation on tumour development are unclear. The TME blocks differentiation of myeloid derived suppressor cells (MDSCs) performing an immunosuppressive functions through, in part, up regulation of nitric oxide synthase (iNOS) which suppresses the T-cell immune response. In these process ROS is likely to play a signalling role in the TME and in communication with the host's surrounding tissue. It has been reported that ROS relates to the RASRAF-MEK-ERK pathway activation promoting tumour proliferation. Also, ROS activates the apoptotic ASK1-JNK-p38 MAPK-Caspase-3 pathway in cancerous and immune cells, which activates TLR-mediated human innate immunity and so inhibits tumour progression. ROS signalling and host immune response are likely to play different roles at the different stages of cancer development: from tumour inhibition by immune surveillance mechanisms to cancer promotion when the tumour adapts to and exploits inflammation microenvironment to a shortcut progression [16].

Crosstalk and communication among cells making up the GI microenvironment discussed briefly above are an area of intensive research and are considered promising targets for the development of novel therapy. To progress this development, the wider application of systems biology integrative approaches may be extremely useful in helping to form our understanding of how this complex GI microenvironment of cell populations interacts, communicates and forms integrated responses to pathogens and diseases. This direction of research also challenges systems biology itself to expand its methodology from an investigation of specific cellular function to the coherent function of different cells which communicate with each other and respond in a concerted manner to pathological stimuli at the population level. A powerful tool in this new systems research landscape is the non-invasive bio photonic techniques of deep-tissue imaging that can help to track the development of complication conditions in the GI tract in real time and image the effects of drug therapy directly during drug administration. Stress imaging using new transgenic mice bearing mutations in specific genes, e.g. relevant to the immune system, may help to elucidate how host immunity modulates tumour growth, and also inflammatory and autoimmune response development.

The collaborative investigation of different research groups focusing on specific cell populations in the GI tract may lead to the design of effective treatment strategies and preventive countermeasures against 
acute GI damage induced by chronic inflammation and GI injury as a result of radiotherapy. Promising directions in this area might be: 1) combination drug therapy when drugs in combination inhibit different signals in the GI microenvironment, 2) adjuvant therapy, and 3) drug repurposing. For example, the research of the gut-microbiotahost immunity cooperation briefly discussed above may result in the development of a novel potential adjuvant therapy where commensal probiotics are used as modulators of the GI microenvironment and adjuvant to radiotherapy. Drug repurposing to modulate the immuneGI-brain communication axis in psychoneuroimmunology and immunobehavioral research might lead to the development of novel therapeutic strategies for functional GI disorders and infection-induced anxiety. In these therapies, ROS signalling pathways are likely to be the key targets for monitoring and drug intervention. Given increasing evidence for the pro- and anti-inflammation roles of ROS signalling, drug modulation of ROS level in the GI tract may be a promising route in the treatment of such diseases as inflammatory bowel disease, cancer, and acute GI injury induced by radiation-GI pathologies against which there are no currently effective therapies. But such therapy requires a deeper understanding of the role of ROS in pro- and anti-inflammation signalling and immune modulation for different GI diseases. Here is where systems biology approaches and personalised medicine approach may be of particular benefit.

The crosstalk among cells making up TME highlighted above may also represent a promising target for innovative anticancer treatments that perturb inter-cell communication in the TME and suppresses tumour development and drug resistance. In this way, novel strategies in personalized cancer therapy may be developed on the basis of repurposing drugs that modulate different cell populations and suppress tumour progression through inhibition of immunosuppressive, proangiogenic, and tissue remodelling factor release in the TMEs.

\section{References}

1. Jathoul AP, Laufer J, Ogunlade O, Treeby B, Cox B, et al. (2015) Deep in vivo photoacoustic imaging of mammalian tissues using a tyrosinase-based genetic reporter. Nat Photonics 9: 239-246.
2. Papineni Rao VL (2015) Radiobioluminescence, Cerenkov luminescence - God light in likes, a potential in radiation therapy imaging. J Cancer Res Ther 11: 241.

3. Chaveroux C, Carraro V, Canaple L, Averous J, Maurin AC, et al. (2015) In vivo imaging of the spatiotemporal activity of the elF2 -ATF4 signalling pathway: Insights into stress and related disorders. Sci Signal 8: rs5.

4. Ahmed I, McLaughlin S, Umar S, Papineni RVL (2014) Therapeutic strategies protect mucin and microbiota affected during early phase of radiation damage or during infection by Citrobacter rodentium. In Procedeedings The 60th Annual Meeting of the Radiation Research Society, Las Vegas: 21-24.

5. Papineni R, Ahmed I, LeVine S, Bradley K, McLaughlin W, et al. (2013) Molecular imaging of early inflammatory response during hyperplasia and/or colitis in response to bacterial infection. Cancer Res 73: 2864.

6. Papineni R, Mor G, McLaughlin W, Holmberg J (2012) Induced myeloperoxidase activity in ovarian cancer. FASEB J 26: 657.

7. Papineni R, Vinicium C, Pizzonia J, Holmberg, J Mor G (2012) Non-invasive determination of in vivo myeloperoxisase activity in ovarian cancer. Cancer Res 72: 5259.

8. Schäppi MG, Jaquet V, Belli DC, Krause KH (2008) Hyper inflammation in chronic granulomatous disease and anti-inflammatory role of the phagocyte NADPH oxidase. Semin Immunopathol 30: 255-271.

9. Umar S (2010) Intestinal stem cells. Curr Gastroenterol Rep 12: 340-348.

10. Leibowitz BJ, Qiu W, Liu H, Cheng T, Zhang L, et al. Uncoupling p53 functions in radiation-induced intestinal damage via PUMA and p21. Mol Cancer Res 9: 616-625.

11. Kamada N, Seo SU, Chen GY, Núñez G (2013) Role of the gut microbiota in immunity and inflammatory disease. Nat Rev Immunol 13: 321-335.

12. Vitetta L, Coulson S, Linnane AW, Butt H (2013) The gastrointestinal micro biome and musculoskeletal diseases: a beneficial role for probiotics and prebiotics. Pathogens 2: 606-626.

13. Papineni R (2014) X-ray irradiation induced bio luminescence: Ex vivo and endoscopic imaging of radio bioluminescence. Cancer Res 74: 4921.

14. Sundman E, Olofsson PS (2014) Neural control of the immune system. Adv Physiol Educ 38: 135-139.

15. Goehler LE, Lyte M, Gaykema RPA (2007) Infection-induced viscero-sensory signals from the gut enhances anxiety: implications for psychoneuroimmunology. Brain Behav Immun 21: 721-726.

16. Conti L, Ruiu R, Barutello G, Macagno M, Bandini S, et al. (2014) Microenvironment, oncoantigens, and antitumor vaccination: lessons learned from BALB-neuT mice. Biomed Res Int. 